\title{
Fußreflexzonenmassage: mehr als ein Placebo?
}

- Forscher aus Taiwan randomisierten 65 Frauen, die nach einer Geburt über Schlafstörungen litten, in zwei Gruppen. Die Verumgruppe erhielt fünf Tage lang täglich eine 30-minütige Fußreflexzonenmassage (FRZM). Die Kontrollgruppe erhielt keine derartige Therapie. Bei Therapieende gaben die Verumpatienten an, besser zu schlafen als die Patienten der Kontrollgruppe. Die Differenz war statistisch signifikant. Die Autoren meinen daher, dass die FRZM eine bei dieser Indikation effektive Therapie sei.

\section{Kommentar}

Dass eine sanfte Fußmassage angenehm ist, entspannt und so den Schlaf in stressvollen
Lebenssituationen fördert, wird wohl kaum jemand bezweifeln. Anhänger der FRZM gehen jedoch sehr viel weiter. Sie glauben, dass sich auf der Fußsohle Reflexzonen befinden, die direkt mit Organsystemen in Verbindung stehen. So wurde dann auch von den Therapeuten in dieser Studie versucht, durch Druck auf "spezifische" Punkte, das Schlafzentrum zu beeinflussen. Da es keine anatomischen Bahnen gibt, die solche Verbindungen herstellen, zählt die FRZM zu den wenig plausiblen Methoden der Alternativmedizin. Studien hierzu sind Mangelware. Daher ist die vorliegende Arbeit es Wert, beachtet zu werden. Leider erlaubt das Studiendesign jedoch keine Aussage über eine spezifische Wirksamkeit der FRZM. Es fehlt jeglicher Versuch, auf unspezifische Therapieeffekte zu kontrollieren. Somit sind die beobachteten Effekte wohl eher durch die wohltuende Entspannung einer Fußmassage zu erklären. Manche werden meinen, das sei letztlich egal, Hauptsache es hilft. Diesem Argument mag ich mich nur teilweise anschließen - denn wenn dem so ist, dann brauchen wir weder den Hokuspokus der Reflexzonen noch speziell ausgebildete FRZM-Therapeuten.

E. ERNST =

\section{- C. Y. Li et al.}

Randomised controlled trial of the effectiveness of using foot reflexology to improve quality of sleep amongst Taiwanese postpartum women. Midwifery 27 (2011) 181-186

\section{Schützt Lebensstiländerung vor Retinopathie?}

\begin{abstract}
Mikrovaskuläre Komplikationen, vor allem retinopathische Veränderungen, kommen bei langjährigen Diabetikern häufig vor. Das Auftreten dieser Komplikationen korreliert eng mit der Glykämie und wird durch Antidiabetika reduziert. Was bewirken Ernährungsumstellung und vermehrte Bewegung?
\end{abstract}

- In die chinesische Da-Qing-Studie wurden 577 Personen mit gestörter Glukosetoleranz (IGT) aufgenommen. Von 1986 bis 1992 wurde durch Ernährungsumstellung, vermehrte Bewegung oder eine Kombination aus beiden die Konversion einer IGT in einen manifesten Diabetes um $40-45 \%$ reduziert.

Zwanzig Jahre nach der Intervention litten in der Kontrollgruppe $90 \%$ an einem manifesten Diabetes, in der Interventionsgruppe waren es $77 \%$. Ein Diabetes manifestierte sich in der Interventionsgruppe 3,6 Jahre später als in der Kontrollgruppe. Der Body-Mass-Index war mit $24,5 \mathrm{~kg} / \mathrm{m}^{2}$ in beiden Gruppen identisch.

Die Inzidenz einer schweren Retinopathie (proliferativ, Photokoagulation,

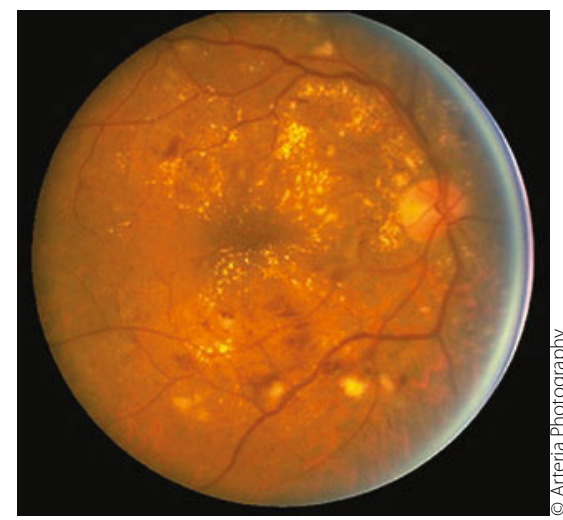

Diabetische Retinopathie: Bewegung und Ernährungsumstellung schützen.

Blindheit) war in der Interventionsgruppe um $47 \%$ niedriger als in der Kontrollgruppe. Eine nicht proliferative Retinopathie stellte sich in der Interventionsgruppe bei 36\% und in der Kontrollgruppe bei 39\% der Patienten ein. Die Entwicklung einer Neuropathie und einer Nephropathie war in beiden Gruppen gleich häufig.

\section{Kommentar}

Erstaunlich ist, dass sich 20 Jahre nach einer Intervention hinsichtlich des Lebensstils
Auswirkungen bei der Entwicklung einer Retinopathie bei Patienten zeigten, die bei Aufnahme in die Studie "nur" eine Störung der Glukosetoleranz aufwiesen. Eine Retinopathie entwickelte sich nur bei den Personen, die manifest diabetisch wurden und diese Krankheit länger als zehn Jahre aufwiesen. Weshalb sah man die positiven Effekte der Lebensstiländerung nur bei der Retinopathie? Zum einen wurde die Untersuchungen zur Nephropathie und Neuropathie 2006 nur bei den Überlebenden durchgeführt; $25 \%$ der Teilnehmer waren inzwischen verstorben. Die Daten zur Retinopathie lagen dagegen aufgrund von Photodokumentationen und Spaltlampenuntersuchungen auch von den Verstorbenen vor. Zum anderen ist denkbar, dass von der Lebensstiländerung unabhängig von einer Blutzuckerreduktion Effekte unbekannter Art mit günstiger Auswirkung aufdie Retinopathie ausgingen. A. WIRTH .

\section{- Q. Gong et al.}

Long-term effects of a randomised trial of a 6-year lifestyle intervention in impaired glucose tolerance on diabetes-related microvascular complications: the China Da Qing Diabetes Prevention Outcome Study. Diabetologia 54 (2011) 300-307 